\title{
Doripenem versus Pseudomonas aeruginosa In Vitro: Activity against Characterized Isolates, Mutants, and Transconjugants and Resistance Selection Potential
}

\author{
Shazad Mushtaq, ${ }^{1}$ Yigong Ge, ${ }^{2}$ and David M. Livermore ${ }^{1 *}$ \\ Antibiotic Resistance Monitoring \& Reference Laboratory, Specialist \& Reference Microbiology Division, \\ Health Protection Agency, Colindale, London, NW9 5HT, United Kingdom, ${ }^{1}$ and \\ Peninsula Pharmaceuticals Inc., Alameda, California $94502^{2}$
}

Received 30 October 2003/Returned for modification 28 January 2004/Accepted 14 April 2004

\begin{abstract}
Doripenem is a broad-spectrum parenteral carbapenem under clinical development in Japan and North America. Its activities against (i) Pseudomonas aeruginosa isolates with graded levels of intrinsic efflux-type resistance, (ii) mutants with various combinations of AmpC and OprD expression, (iii) PU21 transconjugants with class $A$ and $D \beta$-lactamases, and (iv) $P$. aeruginosa isolates with metallo- $\beta$-lactamases were tested by the agar dilution method of the National Committee for Clinical Laboratory Standards. Selection of resistant $P$. aeruginosa mutants was investigated in single- and multistep procedures. Doripenem MICs for isolates without acquired resistance mostly were 0.12 to $0.5 \mu \mathrm{g} / \mathrm{ml}$, whereas meropenem MICs were 0.25 to $0.5 \mu \mathrm{g} / \mathrm{ml}$ and imipenem MICs were 1 to $2 \mu \mathrm{g} / \mathrm{ml}$. The MICs of doripenem, meropenem, ertapenem, and noncarbapenems for isolates with increased efflux-type resistance were elevated, whereas the MICs of imipenem were less affected. The MICs of doripenem were increased by the loss of OprD but not by derepression of AmpC; nevertheless, and as with other carbapenems, the impermeability-determined resistance caused by the loss of OprD corequired AmpC activity and was lost in $\mathrm{OprD}^{-}$mutants also lacking AmpC. The TEM, PSE, PER, and OXA enzymes did not significantly protect $P$. aeruginosa PU21 against the activity of doripenem, whereas MICs of $\geq 16 \mu \mathrm{g} / \mathrm{ml}$ were seen for clinical isolates with VIM and IMP metallo- $\beta$-lactamases. Resistant mutants seemed to be harder to select with doripenem than with other carbapenems (or noncarbapenems), and the fold increases in the MICs were smaller for the resistant mutants. Single-step doripenem mutants were mostly resistant only to carbapenems and had lost OprD; multistep mutants had broader resistance, implying the presence of additional mechanisms, putatively including up-regulated efflux. Most mutants selected with aminoglycosides and quinolones had little or no cross-resistance to carbapenems, including doripenem.
\end{abstract}

Pseudomonas aeruginosa remains a major pathogen and is especially important in critical care and burn units, as well as in patients with cystic fibrosis. Patients with severe pseudomonal infections have a poor prognosis, partly because the organism has considerable inherent resistance, caused by the combination of impermeability, multiple efflux systems, and a chromosomal AmpC $\beta$-lactamase. In addition, $P$. aeruginosa can develop mutational resistance to all the various "antipseudomonal" penicillins, cephalosporins, carbapenems, aminoglycosides, and fluoroquinolones presently available (15).

No potent antipseudomonal drugs have been developed over the last decade, leaving meropenem as the most active antipseudomonal agent available. Doripenem (S-4661) (23) is a new parental carbapenem discovered by Shionogi Ltd. and now being codeveloped, for intravenous use, with Peninsula Pharmaceuticals in North America; phase I trials of a nebulized formulation are also in progress. Like the carbapenems available at present, doripenem has a trans-configured 6-hydroxyethyl group, which protects it against $\beta$-lactamases, and, like meropenem, has some stability against human renal dehydropeptidase I. Doripenem inhibits the growth of nonferment-

* Corresponding author. Mailing address: Antibiotic Resistance Monitoring \& Reference Laboratory, Specialist \& Reference Microbiology Division, Health Protection Agency Colindale, London NW9 5HT, United Kingdom. Phone: 44 (0)20-8327-7223. Fax: 44 (0)208327-6264. E-mail: david.livermore@hpa.org.uk. ers as well as members of the family Enterobacteriaceae, anaerobes, and gram-positive cocci (23). In the present investigation we studied its antipseudomonal activity in detail. This is critical for any carbapenem, not only because carbapenems are among the most powerful antipseudomonal agents, but also because imipenem and meropenem differ in their antipseudomonal behaviors, both from each other and from other $\beta$-lactams (12). Unlike the cephalosporins and piperacillin, neither imipenem nor meropenem is affected by derepression of the AmpC $\beta$-lactamase or by most of the acquired $\beta$-lactamases that occur in the species $(3,15)$; however, imipenem and, to a lesser degree, meropenem are compromised by loss of the carbapenem-specific porin OprD. Meropenem, but not imipenem, is also affected by up-regulation of efflux, although this mechanism rarely confers frank resistance (12). Both imipenem and meropenem are hydrolyzed by the IMP, SPM, and VIM metallo- $\beta$-lactamases now gradually emerging in the species $(16,22)$.

\section{MATERIALS AND METHODS}

Strains. $P$. aeruginosa strains and isolates with graded levels of intrinsic resistance to $\beta$-lactams, mediated by impermeability and efflux, have been collected by the Antibiotic Resistance Monitoring \& Reference Laboratory over many years, as described previously $(3,18,24)$. These organisms show related degrees of cross-resistance or susceptibility to $\beta$-lactams and non- $\beta$-lactams $(10,13)$ and lack constitutive $\beta$-lactamase production. Representative strains were confirmed to retain fully susceptible penicillin-binding proteins and consistent outer membrane protein profiles but to vary in their efflux activities (11). Mucoid strains, 
used for mutant selection, were recent isolates from cystic fibrosis patients. Other strains tested included (i) recent clinical isolates with IMP and VIM metallo- $\beta$ lactamases collected in the United Kingdom, Japan, Canada, and Oman; (ii) clinical and laboratory mutants with various combinations of AmpC and OprD expression (14); and (iii) transconjugants of $P$. aeruginosa PU21 with class A and D $\beta$-lactamases. $P$. aeruginosa ATCC 27853 was used as a general control.

MIC determinations. MICs were determined on Mueller-Hinton agar (Oxoid, Basingstoke, United Kingdom) by the method of the National Committee for Clinical Laboratory Standards (NCCLS) (20). The drugs tested were doripenem (lot no. CF 2066; manufactured by Shionogi \& Co., Ltd., Osaka, Japan), ertapenem and imipenem (Merck, Hoddesdon, United Kingdom), meropenem (AstraZeneca, Macclesfield, United Kingdom), ceftazidime (GlaxoSmithKline, Stevenage, United Kingdom), cefepime (Bristol Myers Squibb, Hounslow, United Kingdom), and piperacillin and tazobactam (Wyeth, Taplow, United Kingdom). Tazobactam was used at $4 \mu \mathrm{g} / \mathrm{ml}$ in combination with piperacillin. Ciprofloxacin (Bayer, Newbury, United Kingdom), levofloxacin (Aventis, Uxbridge, United Kingdom), and carbenicillin and tobramycin (Sigma, Poole, United Kingdom) were used for mutant selection.

Mutant selection. Single-step mutants were selected by plating ca. $5 \times 10^{8}$ CFU, in 100- $\mu$ l volumes, from duplicate overnight broth cultures onto 9-cmdiameter plates with Mueller-Hinton agar containing antibiotics at doubling concentrations from 2 to 16 times the MIC. Any colonies that grew after overnight incubation at $37^{\circ} \mathrm{C}$ were counted. Mutation frequencies were calculated, and the results of duplicate plate counts on antibiotic-free agar were taken as the denominator.

Multistep mutants were selected by growing the bacteria overnight in $10 \mathrm{ml}$ of cation-adjusted Mueller-Hinton broth, initially with the antibiotic at one-half the MIC. One hundred microliters of these cultures was then transferred to fresh broth with double the original antibiotic concentration, and the culture was reincubated overnight. This process was repeated for up to 12 cycles, with the drug concentration doubled after each cycle or until no further increase in resistance could be obtained. The MICs of the selective drugs and other agents were determined after the 3rd, 6th, 9th, and 12th cycles and/or after the last cycle at which growth was obtained.

Serotyping by slide agglutination by the International Antigenic Typing Scheme was used to confirm that the organisms recovered postselection were not contaminants

Molecular characterization of mutants. Outer membrane proteins were extracted from logarithmic-phase cultures with sodium lauroyl sarkosinate and profiled by sodium dodecyl sulfate-polyacrylamide gel electrophoresis by the method of Chart (2). $\beta$-Lactamase specific activities were measured with crude sonicates prepared from cells grown as described above for the outer membrane protein studies; $0.1 \mathrm{mM}$ nitrocefin in $0.1 \mathrm{M}$ phosphate buffer $(\mathrm{pH} 7.0$ ) was the assay substrate, with activity measured by spectrophotometry at $482 \mathrm{~nm}$ at $37^{\circ} \mathrm{C}$ and standardized against the protein concentration.

\section{RESULTS}

Activity against isolates with intrinsic resistance. Isolates were graded, based on previous results, as supersusceptible to carbenicillin (MIC, $\leq 16 \mu \mathrm{g} / \mathrm{ml}$ ), normally susceptible (MIC, 32 to $128 \mu \mathrm{g} / \mathrm{ml}$ ), resistant (MIC $256 \mu \mathrm{g} / \mathrm{ml}$ ), and highly resistant $(\mathrm{MIC} \geq 512 \mu \mathrm{g} / \mathrm{ml}$ ). The geometric mean MICs of doripenem and its comparators were then calculated for each of these groups. The MICs of doripenem were lower for the carbenicillin-supersusceptible isolates than for the normally susceptible isolates and were increased for the carbenicillinresistant isolates and, especially, the highly resistant isolates (Fig. 1). Similar relationships were seen for meropenem, ertapenem, and noncarbapenem $\beta$-lactams; but the relationship for imipenem was weak. Within each category, except the carbenicillin-supersusceptible group, the MICs of doripenem were lower than those of any other $\beta$-lactam tested, although the differential compared with the MIC of meropenem was less than 1 doubling dilution.

Activities against OprD and AmpC mutants. The MICs for the two mutant series are shown in Table 1: the 1405 series of strains originated from an $\mathrm{AmpC}$-derepressed (AmpC-CON)

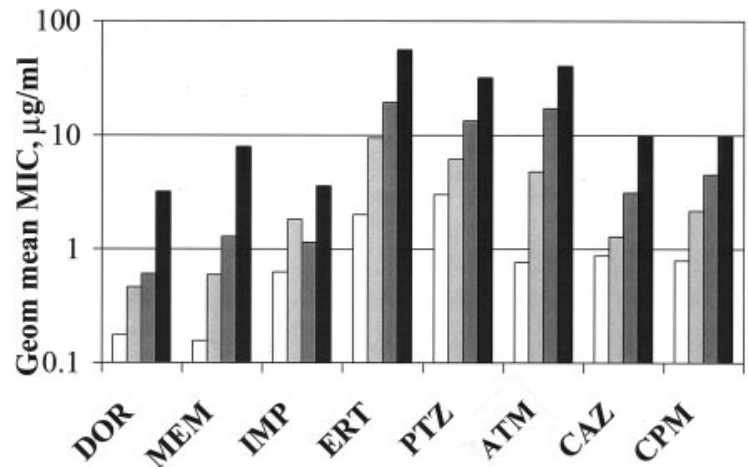

FIG. 1. Geometric mean MICs of antibiotics for isolates sorted by their levels of intrinsic resistance to carbenicillin: white, carbenicillin MIC, $\leq 16 \mu \mathrm{g} / \mathrm{ml}$; light grey, carbenicillin MIC, 32 to $128 \mu \mathrm{g} / \mathrm{ml}$; dark grey, carbenicillin MIC, $256 \mu \mathrm{g} / \mathrm{ml}$; black, carbenicillin MIC, $\geq 512 \mu \mathrm{g} /$ ml. Abbreviations: DOR, doripenem; MEM, meropenem; IMP, imipenem, ERT, ertapenem; PTZ, piperacillin-tazobactam, as tested with a fixed concentration of $4 \mu \mathrm{g}$ of tazobactam per ml; ATM, aztreonam; CAZ, ceftazidime; CPM, cefepime.

isolate, from which AmpC-basal (AmpC-DEF) and OprD mutants were derived (14). The 1405 AmpC-CON OprD organism was used to derive an AmpC-DEF OprD ${ }^{-}$mutant that lacked both the $\beta$-lactamase and the porin. Derivation in the 2297 series of organisms was similar, except that a parent, pretherapy, AmpC-inducible, $\mathrm{OprD}^{+}$isolate was also available.

Comparison of the MICs for strains 2297 AmpC-IND and 2297 AmpC-CON showed that derepression of AmpC in an $\mathrm{OprD}^{+}$background was associated, at most, with single-dilution increases in carbapenem MICs, including that of doripenem, whereas the MICs of noncarbapenem $\beta$-lactams were increased $\geq 16$-fold. Loss of AmpC (compare the MICs for the AmpC-CON OprD ${ }^{+}$and AmpC-DEF OprD ${ }^{+}$organisms) increased the levels of susceptibility to the carbapenems, including doripenem, two- to eightfold and restored full susceptibility to the noncarbapenems, confirming earlier results (14). Loss of OprD from the AmpC-CON organisms increased the MICs of doripenem and the other carbapenems but had no effect on the MICs of noncarbapenems; loss of AmpC from these OprD mutants markedly reduced the MICs of the carbapenems (except that of meropenem for the 2297 series of organisms), indicating that resistance corequired $\mathrm{AmpC}$ as well as the impermeability caused by the porin loss.

Activity against $\boldsymbol{P}$. aeruginosa PU21 transconjugants. None of the class A and D $\beta$-lactamases introduced into strain PU21 caused a substantial increase in the carbapenem MICs, although 1- to 2-dilution effects were seen for transconjugants with several OXA-10-related enzymes, including OXA-10 itself and OXA-14 (Table 1). These effects were least evident with imipenem and were stronger with doripenem, meropenem, and ertapenem. Extended-spectrum class A (PER-1) and D (OXA-11, -14, -16, -101, and -103) enzymes conferred resistance to all the cephalosporins, aztreonam, and piperacillintazobactam. All the enzymes conferred protection against piperacillin-tazobactam in $P$. aeruginosa .

Activity against metallo- $\boldsymbol{\beta}$-lactamase producers. The MICs for $P$. aeruginosa isolates with metallo- $\beta$-lactamases are shown in Table 1. Except for one IMP-7 producer (P/6330), these 
TABLE 1. MICs for P. aeruginosa AmpC and OprD mutants, PU21 transconjugants, and isolates with metallo- $\beta$-lactamases ${ }^{a}$

\begin{tabular}{|c|c|c|c|c|c|c|c|c|}
\hline \multirow{2}{*}{ Strain ( $\beta$-lactamase, source) } & \multicolumn{8}{|c|}{$\operatorname{MIC}(\mu \mathrm{g} / \mathrm{ml})$} \\
\hline & DOR & MEM & IMP & ERT & PTZ & ATM & CAZ & CPM \\
\hline \multicolumn{9}{|c|}{ AmpC and OprD expression mutants of $P$. aeruginosa 1405 and 2297} \\
\hline 2297 AmpC-IND OprD ${ }^{+}$ & 0.25 & 0.25 & 2 & 4 & 4 & 4 & 1 & 2 \\
\hline 2297 AmpC-CON OprD $^{+}$ & 0.5 & 0.5 & 1 & 8 & $>64$ & 64 & 64 & 32 \\
\hline 2297 AmpC-DEF OprD ${ }^{+}$ & 0.06 & 0.125 & 0.25 & 2 & 4 & 4 & 1 & 2 \\
\hline 2297 AmpC-CON OprD ${ }^{-}$ & 8 & 8 & 16 & 64 & $>64$ & 64 & 64 & 32 \\
\hline 2297 AmpC-DEF OprD ${ }^{-}$ & 1 & 4 & 2 & 16 & 8 & 4 & 2 & 2 \\
\hline 1405 AmpC-CON OprD ${ }^{+}$ & 0.5 & 0.5 & 2 & 16 & $>64$ & 64 & 64 & 32 \\
\hline 1405 AmpC-DEF OprD ${ }^{+}$ & 0.125 & 0.25 & 0.5 & 4 & 8 & 16 & 2 & 8 \\
\hline 1405 AmpC-CON OprD ${ }^{-}$ & 8 & 16 & 16 & $>64$ & $>64$ & 64 & 64 & 32 \\
\hline 1405 AmpC-DEF OprD ${ }^{-}$ & 0.06 & 0.125 & 0.25 & 2 & 8 & 8 & 2 & 4 \\
\hline \multicolumn{9}{|l|}{ P. aeruginosa $\mathrm{PU} 21$ and transconjugants } \\
\hline PU21R ${ }^{-}$ & 1 & 1 & 2 & 16 & 16 & 8 & 2 & 2 \\
\hline PU21/TEM-2 & 2 & 2 & 2 & 32 & $>64$ & 4 & 16 & 8 \\
\hline PU21/PSE-1 & 2 & 2 & 4 & 32 & 16 & $>64$ & $>64$ & 32 \\
\hline PU21/PSE-3 & 2 & 1 & 2 & 16 & $>64$ & 8 & 2 & 4 \\
\hline PU21/PSE-4 & 2 & 2 & 2 & 4 & $>64$ & 8 & 2 & 2 \\
\hline PU21/PER-1 & 1 & 1 & 2 & 16 & 8 & 4 & 1 & 4 \\
\hline PU21/OXA-2 & 0.5 & 0.5 & 2 & 8 & 8 & 2 & 16 & 2 \\
\hline PU21/OXA-10-low & 4 & 4 & 4 & 64 & $>64$ & 32 & 16 & 32 \\
\hline PU21/OXA-10 high & 8 & 4 & 2 & 32 & $>64$ & 8 & 64 & 16 \\
\hline PU21/OXA-11 & 1 & 2 & 4 & 16 & 64 & 32 & $>64$ & $>64$ \\
\hline PU21/OXA-14 & 4 & 4 & 2 & 32 & $>64$ & 16 & $>64$ & 64 \\
\hline PU21/OXA-15 & 2 & 2 & 2 & 32 & 32 & 8 & $>64$ & 16 \\
\hline PU21/OXA-16 & 1 & 1 & 2 & 16 & 16 & 4 & 64 & 16 \\
\hline PU21/OXA-M101 & 1 & 1 & 2 & 16 & 16 & 8 & 32 & 8 \\
\hline PU21/OXA-M103 & 1 & 2 & 2 & 32 & $>64$ & 8 & 64 & 16 \\
\hline PU21/NPS-1 & 4 & 4 & 2 & 32 & $>64$ & 8 & 2 & 8 \\
\hline \multicolumn{9}{|l|}{$P$. aeruginosa isolates with metallo- $\beta$-lactamases } \\
\hline 101/1477 (IMP-1, Japan) & $>64$ & $>64$ & $>64$ & $>64$ & $>64$ & 8 & $>64$ & $>64$ \\
\hline ACRS35 (VIM-2, UK) & 16 & 16 & 32 & $>64$ & 32 & 8 & 32 & 16 \\
\hline A2 (VIM-?, UK) & $>64$ & $>64$ & $>64$ & $>64$ & $>64$ & 16 & $>64$ & 64 \\
\hline Oman (VIM-?, Oman) & 32 & 32 & $>64$ & $>64$ & $>64$ & 8 & $>64$ & $>64$ \\
\hline ACRS10 (VIM-10, UK) & $>64$ & $>64$ & $>64$ & $>64$ & $>64$ & 64 & 64 & 64 \\
\hline ACRS22 (VIM-4, UK) & 32 & 64 & $>64$ & $>64$ & $>64$ & 16 & 64 & 64 \\
\hline ACRS30 (VIM-4, UK) & 64 & 64 & $>64$ & $>64$ & $>64$ & 8 & 32 & 64 \\
\hline ACRS29 (VIM-4, UK) & $>64$ & $>64$ & $>64$ & $>64$ & $>64$ & 16 & 64 & $>64$ \\
\hline ACRS28 (VIM-2, UK) & 16 & 16 & 32 & $>64$ & 32 & 8 & 32 & 16 \\
\hline ACRS31 (VIM-2, UK) & 32 & 64 & $>64$ & $>64$ & $>64$ & 64 & 32 & 16 \\
\hline ACRS32 (VIM-2, UK) & 64 & 64 & $>64$ & $>64$ & $>64$ & 64 & 64 & 16 \\
\hline P/6327 (IMP-7, Canada) & $>64$ & $>64$ & $>64$ & $>64$ & 16 & 16 & $>64$ & $>64$ \\
\hline P/6328 (IMP-7, Canada) & $>64$ & $>64$ & $>64$ & $>64$ & 32 & 16 & $>64$ & $>64$ \\
\hline P/6329 (IMP-7, Canada) & $>64$ & $>64$ & $>64$ & $>64$ & 32 & 16 & $>64$ & $>64$ \\
\hline P/6330 (IMP-7, Canada) & 4 & 16 & 16 & 64 & 16 & 16 & 2 & 8 \\
\hline
\end{tabular}

${ }^{a}$ Abbreviations: CON, derepressed, expressing AmpC copiously without induction; DEF, basal, expressing only trace AmpC even with induction; IND, AmpC inducible; UK, United Kingdom; DOR, doripenem; MEM, meropenem; IMP, imipenem, ERT, ertapenem; PTZ, piperacillin-tazobactam, as tested with a fixed concentration of $4 \mu \mathrm{g}$ of tazobactam per ml; ATM, aztreonam; CAZ, ceftazidime; CPM, cefepime.

organisms were unequivocally resistant to doripenem and other carbapenems; $\mathrm{P} / 6330$ had reduced susceptibility to doripenem and was resistant to the other carbapenems. The metallo- $\beta$-lactamase producers were also broadly resistant to noncarbapenems, except that 12 of the 15 had moderately retained susceptibilities (MICs, 8 to $16 \mu \mathrm{g} / \mathrm{ml}$ ) to aztreonam, and $\mathrm{P} / 6330$ (again) appeared to be susceptible to ceftazidime.

Single-step mutant selection. Mutant selection was performed as a single-step procedure for eight $P$. aeruginosa strains. Four strains (strains A, B, C, and D) were carbapenem susceptible and nonmucoid, three strains (strains E, F, and G) were carbapenem susceptible and mucoid, and one strain (strain $\mathrm{H}$ ) was nonmucoid and had an $\mathrm{OprD}^{-}$phenotype. One mucoid strain (strain $\mathrm{G}$ ) was supersusceptible to penicillins, cephalosporins, and aztreonam; all the others (including the $\mathrm{OprD}^{-}$organism, strain $\mathrm{H}$ ) had normal levels of susceptibility to these drugs, typical of those for the species as a whole.

The abilities of the antibiotics to select mutants is summarized in Table 2. Mutant selection with doripenem occurred with fewer of the strains than with the other compounds, and the final multiple of the MIC achieved was lower for doripenem than for other agents, never exceeding quadruple the starting MIC. By contrast, ceftazidime, for example, selected singlestep mutants for which the MICs increased up to 32-fold. The patterns of the increases in the MICs for the mutants selected with particular antibiotics mostly showed considerable consistency among the different strains, and the geometric mean fold increases are therefore shown in Table 2 as summary param- 
TABLE 2. Isolation and phenotypes of single-step $P$. aeruginosa mutants ${ }^{a}$

\begin{tabular}{|c|c|c|c|c|c|c|c|c|c|c|c|c|c|c|}
\hline \multirow{2}{*}{$\begin{array}{l}\text { Selective } \\
\text { agent }\end{array}$} & \multicolumn{4}{|c|}{$\begin{array}{l}\text { No. of strains (of } 8 \text { ) giving } \\
\text { mutants at MIC multiple of: }\end{array}$} & \multirow{2}{*}{$\begin{array}{l}\text { Total no. } \\
\text { of mutants } \\
\text { examined }\end{array}$} & \multirow{2}{*}{$\begin{array}{l}\text { No. of source } \\
\text { strains for } \\
\text { mutants }\end{array}$} & \multicolumn{8}{|c|}{ Geometric mean fold increase in MIC } \\
\hline & $2 \times$ & $4 \times$ & $8 \times$ & $16 \times$ & & & DOR & IMP & MEM & ERT & ATM & PTZ & CAZ & CFM \\
\hline Doripenem & 3 & 1 & 0 & 0 & 30 & 4 & 6.5 & 9.9 & 10.6 & 4.1 & 1.4 & 1.5 & 1.4 & 1.0 \\
\hline Imipenem & 6 & 2 & 3 & 0 & 58 & 6 & 4.3 & 6.4 & 5.7 & 2.7 & 1.3 & 1.6 & 1.2 & 1.0 \\
\hline Meropenem & 7 & 7 & 3 & 0 & 42 & 5 & 3.5 & 4.8 & 5.0 & 2.8 & 1.3 & 1.7 & 1.3 & 1.2 \\
\hline Ertapenem & 6 & 3 & 1 & 1 & 40 & 6 & 4.9 & 6.0 & 8.1 & 5.9 & 1.3 & 1.3 & 1.4 & 1.2 \\
\hline Carbenicillin & 7 & 6 & 2 & 0 & 61 & 7 & 1.3 & 1.0 & 2.1 & 1.7 & 8.3 & 3.1 & 3.7 & 3.2 \\
\hline Ceftazidime & 8 & 8 & 6 & 0 & 73 & 8 & 1.2 & 0.9 & 1.2 & 1.2 & 5.5 & 9.9 & 9.1 & 4.4 \\
\hline Ciprofloxacin & 6 & 3 & 1 & 0 & 67 & 7 & $1.1^{b}$ & $1.4^{b}$ & $1.2^{b}$ & $0.9^{b}$ & 0.8 & 1.0 & 0.9 & 1.1 \\
\hline Tobramycin & 7 & 2 & 1 & 1 & 46 & 7 & 0.7 & 0.8 & 0.8 & 0.7 & 1.5 & 1.2 & 1.6 & 1.4 \\
\hline
\end{tabular}

${ }^{a}$ Abbreviations: DOR, doripenem; MEM, meropenem; IMP, imipenem, ERT, ertapenem; PTZ, piperacillin-tazobactam, as tested with a fixed concentration of $4 \mu \mathrm{g}$ of tazobactam per ml; ATM, aztreonam; CAZ, ceftazidime; CPM, cefepime.

${ }^{b}$ The MICs of carbapenem, but not those of the other $\beta$-lactams tested, were raised for most ciprofloxacin-selected mutants of strain D; for the MICs of carbapenems and other $\beta$-lactams mutants of other strains mostly showed only twofold (i.e., insignificant) upward or downward shifts. The exceptional behavior of strain D is hidden in the calculation of the geometric means.

eters. For most mutants selected with carbapenems, including doripenem, and all those selected with imipenem only, the MICs of carbapenems, not other $\beta$-lactams, were increased by fourfold or more. Occasional exceptions to this generalization, particularly among the mutants selected with meropenem and among the mutants of strain $\mathrm{G}$ selected with ertapenem, were organisms with broader cross-resistance, including to noncarbapenems as well as carbapenems.

For mutants selected with ceftazidime, the MICs of ceftazidime consistently increased fourfold or more, as did those of cefepime, aztreonam, and piperacillin-tazobactam, but, except for one mutant of strain G, the increases in the MICs of any carbapenem never exceeded twofold. Mutants selected with carbenicillin were more diverse. Almost without exception the increases in the MICs of the cephalosporins, aztreonam, and piperacillin-tazobactam were at least two- or fourfold; increases in the MICs of doripenem, ertapenem, and meropenem of two- to (occasionally) fourfold were also detected for many strains, although such increases in the MIC of imipenem were detected only for mutants of strain G. The MICs for mutants selected with ciprofloxacin varied by strain. Twofold or (rarely) fourfold upward or downward shifts in the MICs of $\beta$-lactams were detected for virtually all mutants selected from strains A, B, C, F, G, and H. Two- to eightfold increases in the MICs of carbapenems were mostly detected for mutants selected from one nonmucoid strain (strain D), but increases in the MICs of cephalosporins and aztreonam were not detected. Only minor shifts in $\beta$-lactam MICs for the mutants selected with tobramycin were detected: often twofold increases for noncarbapenems and twofold decreases for carbapenems. The MIC of doripenem never exceeded $8 \mu \mathrm{g} / \mathrm{ml}$ for any single-step mutant, whereas the maximum MICs of imipenem, meropenem, and ceftazidime were 16,32 , and $64 \mu \mathrm{g} / \mathrm{ml}$, respectively.

Multistep mutant selection. Strains A, B, G, and $\mathrm{H}$ were used for multistep selection, which was continued for up to 12 cycles. In practice, however, the maximum achievable levels of resistance were reached after five to seven cycles. Multistep mutants mostly (and unsurprisingly) showed broader crossresistance than single-step mutants. The increases in the carbapenem MICs were eightfold or greater for all six mutants selected with doripenem after three to five cycles; and those of cefepime, ceftazidime, aztreonam, and piperacillin-tazobactam
TABLE 3. MICs for mutants after repeated selection cycles with doripenem ${ }^{a}$

\begin{tabular}{|c|c|c|c|}
\hline \multirow{2}{*}{ Strain and drug } & \multicolumn{3}{|c|}{ MIC $(\mu \mathrm{g} / \mathrm{ml})$ after selection cycle: } \\
\hline & 0 & 3 & 5 \\
\hline \multicolumn{4}{|l|}{ Strain A } \\
\hline DOR & 0.5 & 16 & 32 \\
\hline IMP & 2 & 16 & 32 \\
\hline MEM & 0.5 & 16 & 32 \\
\hline ERT & 8 & 128 & $>128$ \\
\hline ATM & 1 & 4 & 8 \\
\hline PTZ & 2 & 16 & 8 \\
\hline CAZ & 2 & 4 & 2 \\
\hline CPM & 2 & 4 & 16 \\
\hline \multicolumn{4}{|l|}{ Strain $\mathrm{G}$} \\
\hline DOR & 0.125 & 4 & 8 \\
\hline IMP & 2 & 16 & 16 \\
\hline MEM & 0.125 & 4 & 4 \\
\hline ERT & 0.5 & 64 & 64 \\
\hline ATM & 0.5 & 2 & 4 \\
\hline PTZ & 4 & 8 & 16 \\
\hline CAZ & 0.5 & 4 & 8 \\
\hline CPM & 0.125 & 8 & 16 \\
\hline \multicolumn{4}{|l|}{ Strain B } \\
\hline DOR & 0.5 & 8 & 32 \\
\hline IMP & 2 & 32 & 32 \\
\hline MEM & 0.5 & 16 & 64 \\
\hline ERT & 16 & 128 & $>128$ \\
\hline ATM & 1 & 8 & 16 \\
\hline PTZ & 2 & 64 & 64 \\
\hline CAZ & 4 & 16 & 16 \\
\hline CPM & 4 & 4 & 32 \\
\hline
\end{tabular}

Strain $\mathrm{H}^{b}$

$\begin{array}{lr}\text { DOR } & 8 \\ \text { IMP } & 16 \\ \text { MEM } & 4 \\ \text { ERT } & 128 \\ \text { ATM } & 8 \\ \text { PTZ } & 64 \\ \text { CAZ } & 8 \\ \text { CPM } & 4\end{array}$

${ }^{a}$ Abbreviations: DOR, doripenem; MEM, meropenem; IMP, imipenem, ERT, ertapenem; PTZ, piperacillin-tazobactam, as tested with a fixed concentration of $4 \mu \mathrm{g}$ of tazobactam; per ml; ATM, aztreonam; CAZ, ceftazidime; CPM, cefepime. Strains A and B were phenotypically typical $P$. aeruginosa strains, strain $G$ was a mucoid strain supersusceptible to $\beta$-lactams, and strain $H$ was already resistant to carbapenems, owing to the absence of OprD.

${ }^{b}$ No mutants with increased carbapenem resistance were obtained from this strain, which began as resistant, with an $\mathrm{OprD}^{-}$phenotype. 
TABLE 4. Characterization of doripenem-selected mutants

\begin{tabular}{|c|c|c|c|c|c|c|c|c|c|c|c|}
\hline \multirow{2}{*}{ Strain code } & \multirow{2}{*}{ Selective conditions } & \multicolumn{8}{|c|}{$\operatorname{MIC}(\mu \mathrm{g} / \mathrm{ml})^{a}$} & \multirow{2}{*}{$\begin{array}{l}\text { Sp act vs nitrocefin } \\
\text { (nmol/min/mg of } \\
\text { protein) }\end{array}$} & \multirow{2}{*}{$\mathrm{OprD}^{b}$} \\
\hline & & DOR & ERT & IMP & MEM & CAZ & СРM & PTZ & ATM & & \\
\hline Parent A & & 0.5 & 8 & 2 & 0.5 & 1 & 2 & 2 & 2 & 118 & + \\
\hline A/DORI/8/C3 & $\begin{array}{l}\text { Strain A, multistep, three cycles, } \\
8 \mu \mathrm{g} \text { of doripenem per ml }\end{array}$ & 16 & 128 & 16 & 16 & 4 & 4 & 16 & 4 & 169 & - \\
\hline Parent B & & 0.5 & 16 & 2 & 0.5 & 1 & 2 & 4 & 4 & 160 & + \\
\hline B1/DORI/X4/1 & $\begin{array}{l}\text { Strain B, single step at } 4 \times \text { MIC } \\
\text { of doripenem }\end{array}$ & 4 & 64 & 8 & 4 & 4 & 4 & 8 & 8 & 101 & + \\
\hline B/DORI/16/C5 & $\begin{array}{l}\text { Strain B, multistep, five cycles, } \\
16 \mu \mathrm{g} \text { of doripenem per } \mathrm{ml}\end{array}$ & 32 & $>128$ & 32 & 64 & 16 & 32 & 64 & 16 & 562 & - \\
\hline Parent D & & 0.5 & 16 & 1 & 1 & 4 & 4 & 8 & 16 & 169 & + \\
\hline D2/DORI/X2/3 & $\begin{array}{l}\text { Strain D, single step at } 2 \times \text { MIC } \\
\text { of doripenem }\end{array}$ & 4 & 64 & 16 & 8 & 4 & 4 & 16 & 16 & 160 & - \\
\hline D2/DORI/X2/5 & $\begin{array}{l}\text { Strain D, single step at } 2 \times \text { MIC } \\
\text { of doripenem }\end{array}$ & 4 & 64 & 16 & 8 & 4 & 4 & 16 & 16 & 152 & - \\
\hline
\end{tabular}

${ }^{a}$ Abbreviations: DOR, doripenem; MEM, meropenem; IMP, imipenem, ERT, ertapenem; PTZ, piperacillin-tazobactam, as tested with a fixed concentration of 4 $\mu \mathrm{g}$ of tazobactam per ml; ATM, aztreonam; CAZ, ceftazidime; CPM, cefepime.

${ }^{b}$ See Fig. 2.

were also increased (Table 3). These mutants were of the same serotypes as their parent organisms, discounting any possibility that they were contaminants that had entered the culture during the selection procedure. Similar cross-resistance profiles were seen for mutants selected with meropenem, whereas for mutants selected with imipenem, carbapenem MICs increased four- to eightfold, but, with rare exceptions, the increases in the MICs of the other $\beta$-lactams were only twofold, at most. The MICs of the cephalosporins, aztreonam, piperacillin-tazobactam, and, in most cases, ertapenem increased eightfold or greater for multistep mutants selected with ceftazidime, whereas the increases in the MICs of the carbapenems were mostly only twofold. The MICs of all carbapenem and noncarbapenem drugs except imipenem mostly increased eightfold for the multistep mutants selected with carbenicillin; for imipenem, however, the increases in the MICs were twofold or less except with mutants of strain G. The MIC profiles for multistep mutants selected with levofloxacin and tobramycin showed a diverse scatter, but most mutants had little or no cross-resistance to the $\beta$-lactams, including doripenem.

Irrespective of the selective agent and the number of cycles, we could not drive doripenem or imipenem MICs above 32 $\mu \mathrm{g} / \mathrm{ml}$, whereas meropenem MICs reached $64 \mu \mathrm{g} / \mathrm{ml}$ and those of ertapenem, ceftazidime, cefepime, aztreonam, and piperacillin could be pushed above $128 \mu \mathrm{g} / \mathrm{ml}$.

Profiling of mutants selected with doripenem. Outer membrane proteins and $\beta$-lactamase production were investigated for three single-step and two multistep mutants selected with doripenem and, as a control, one single-step mutant selected with imipenem. The MICs for these organisms are shown in Table 4. Among the single-step mutants, two (D2/DORI/X2/3 and D2/DORI/X2/5) were cross-resistant only to carbapenems, whereas small but consistent increases in the MICs of the noncarbapenems were seen for the third mutant (B1/DORI/ X4/1). The first two organisms mentioned above and the control mutant selected with imipenem were found to have lost OprD (Fig. 2), whereas this protein was retained by B1/DORI/ $\mathrm{X} 4 / 1$. The loss of OprD was also apparent in the two multistep mutants profiled, although these organisms almost certainly had additional mechanisms. None of the single-step mutants showed increased levels of $\beta$-lactamase expression, although one of the multistep mutants showed a small increase in the level of $\beta$-lactamase expression, measured as specific activity (Table 4).

\section{DISCUSSION}

Doripenem resembled meropenem in its activity and behavior, with the MICs of these two carbapenems being identical for most strains or 1 dilution lower for doripenem. Both agents were more active than any other antipseudomonal drug tested. Nevertheless, both drugs had reduced activities against isolates with increased intrinsic resistance, as did all the other compounds tested except imipenem. This is consistent with the view that doripenem, like meropenem, is affected by efflux, whereas imipenem largely escapes this mechanism $(9,12)$. A previous study by Masuda and colleagues (19) also suggested

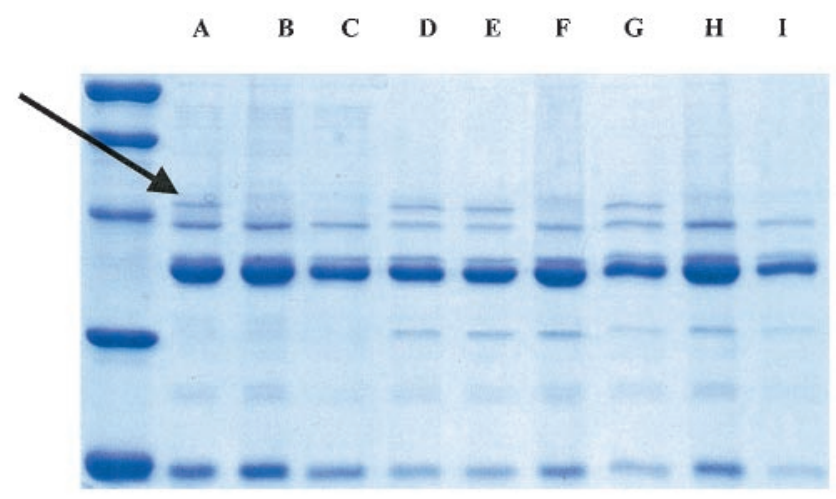

FIG. 2. Outer membrane protein profiles for doripenem-selected mutants. Lanes: A, parent strain A; B, A/DORI/8/C3; C, A1/IMI/X8/1 (imipenem-selected control lacking $\mathrm{OprD}$ ); D, parent strain $\mathrm{B}$; E, B1/DORI/X4/1; F, B/DORI/16/C5; G, parent strain D; H, D2DORI/ $\mathrm{X} 2 / 3$; I, D2/DORI/X2/5. The molecular weights of the markers in the left-most lane are 96,000, 68,000, 45,000, 31,000, and 24,500, from top to bottom, respectively. The arrow indicates OprD. 
that doripenem is a substrate for efflux from $P$. aeruginosa, reinforcing the present conclusion. Although avoidance of efflux is an advantage for imipenem, this must be set against its relatively higher MICs and the fact that simple mutational loss of OprD confers resistance, with this resistance frequently emerging during imipenem therapy. By contrast, multiple mechanisms (efflux plus impermeability) are needed for clear resistance to meropenem and doripenem, and these combinations of mechanisms seem less likely to be selected readily in vivo $(1,12)$.

The loss of OprD increased the doripenem MICs (Table 1), suggesting that, like other carbapenems, doripenem uses this porin to enter the pseudomonal cell. Nevertheless, resistance among $\mathrm{OprD}^{-}$organisms also required functional $\mathrm{AmpC}$ and was lost in AmpC-DEF mutants, as was also found previously for imipenem (14). These data suggest that some slow doripenem hydrolysis occurs under periplasmic conditions; and the implication, not yet exploited, is that a completely stable carbapenem analogue would be little affected by the loss of OprD or that a carbapenem might be made fully active against $\mathrm{OprD}^{-}$mutants by combining it with an inhibitor of AmpC (17).

Like other carbapenems, doripenem lost activity against isolates with IMP and VIM enzymes. Although still uncommon, the gradual spread of these class B metallo- $\beta$-lactamases is a concern. Until 1997, 12 years after the launch of imipenem, only IMP-1 was known and had been found almost exclusively in Japan. Subsequently, the list of IMP variants has grown to 16, with at least 10 VIM types and 1 SPM type also described and with these enzymes recorded-mostly from $P$. aeruginosa-in every populated continent except Australia $(16,22)$. One outbreak of a serotype $012 P$. aeruginosa strain with a VIM carbapenemase in Greece persisted for over 3 years and involved over 200 isolates (16).

In contrast to class B metallo- $\beta$-lactamases, the transfer of various class $A$ and D (serine) $\beta$-lactamases into strain PU21 had little effect on the doripenem MICs, although small increases were seen with OXA-10 and its variants. Similar effects were seen with meropenem and ertapenem, and these results are at variance with earlier results for these comparator carbapenems (4-8). This difference may reflect the use here of Mueller-Hinton agar and the NCCLS methodology, whereas earlier studies used Iso-Sensitest and Diagnostic Sensitivity Test agars with slightly smaller inocula. The significance is unclear, and in any event, OXA-10 variants are rare and largely confined to Turkey.

As with other antipseudomonal drugs, in vitro resistance to doripenem could be selected. Nevertheless, the frequency of mutants resistant to two to four times the starting MIC was lower for doripenem than for the other carbapenems and noncarbapenems tested, and single-step mutants for which increases in MICs were eightfold or greater could not be selected from any of eight strains investigated. The other carbapenems selected mutants from among more of the test strains, and several noncarbapenems, notably including ceftazidime, selected single-step mutants for which increases in MICs were up to 32-fold. Doripenem predominantly selected OprD ${ }^{-}$mutants, the MICs for which were mostly 4 to $8 \mu \mathrm{g} / \mathrm{ml}$; but, like meropenem and unlike imipenem, it also had some propensity to select other mutant phenotypes with broader low-level resis- tance (e.g., mutant B1/DORI/X4/1 [Table 4]). The latter organisms probably had some form of up-regulated efflux. $\mathrm{Mu}-$ tants with this phenotype were predominantly selected by carbenicillin, a compound well known to select for efflux-type variants (21). Whereas most of these mutant selection results were predictable, the studies did give some surprises, notably, the propensity of strain $\mathrm{G}$, with a very low level of intrinsic resistance to $\beta$-lactams, to yield variants selected with carbapenem with broad-spectrum resistance rather than OprD ${ }^{-}$ phenotypes. It is unclear whether the phenomenon was peculiar to strain $G$ or is representative of other strains with low levels of intrinsic resistance. It is likewise unclear why ciprofloxacin tended to select mutants with cross-resistance to carbapenems - putatively $n f x C$ or mexT types (15) - from strain D but not from the other organisms.

Most of the multistep mutants selected with doripenem or meropenem had cross-resistance to noncarbapenem $\beta$-lactams as well as to imipenem and the other carbapenems, implying that efflux was a component in their behavior, along with the loss of OprD. Those mutants selected with imipenem, by contrast, were mostly resistant only to carbapenems, supporting the view that the selectivity of this agent is almost exclusively for OprD ${ }^{-}$mutants. Although fluoroquinolones and even aminoglycosides can select for efflux types in $P$. aeruginosa or, in the case of fluoroquinolones, can select for mexT ( $n f x C)$ mutants, which have up-regulated MexEF-OprN efflux and downregulated OprD, few of the levofloxacin or tobramycin mutants showed any cross-resistance to any carbapenem.

In summary, the behavior of doripenem greatly resembled that of meropenem, but a few differences may be significant. First, doripenem MICs tended to be lower than those of meropenem for strains with elevated levels of intrinsic resistance, and doripenem seemed to have a lesser propensity to select for resistant mutants than meropenem, perhaps because such mutants tended to be slightly less resistant to doripenem than to meropenem. Whether or not these differences translate into any significant advantage will be revealed only by comprehensive clinical investigations.

\section{REFERENCES}

1. Carmeli, Y., N. Troillet, G. M. Eliopoulos, and M. H. Samore. 1999. Emergence of antibiotic-resistant Pseudomonas aeruginosa: comparison of risks associated with different antipseudomonal agents. Antimicrob. Agents Chemother. 43:1379-1382

2. Chart, H. 1994. Sodium dodecyl sulfate polyacrylamide gel electrophoresis for the separation and resolution of bacterial components, p. 21-33. In H. Chart (ed.), Methods in practical laboratory methodology, CRC Press, Inc., Boca Raton, Fla.

3. Chen, H. Y., and D. M. Livermore. 1994. In-vitro activity of biapenem, compared with imipenem and meropenem, against Pseudomonas aeruginosa strains and mutants with known resistance mechanisms. J. Antimicrob. Chemother. 33:949-958.

4. Danel, F., L. M. Hall, D. Gur, H. E. Akalin, and D. M. Livermore. 1995. Transferable production of PER-1 $\beta$-lactamase in Pseudomonas aeruginosa. J. Antimicrob. Chemother. 35:281-294.

5. Danel, F., L. M. Hall, D. Gur, and D. M. Livermore. 1995. OXA-14, another extended-spectrum variant of OXA-10 (PSE-2) $\beta$-lactamase from Pseudomonas aeruginosa. Antimicrob. Agents Chemother. 39:1881-1884.

6. Danel, F., L. M. Hall, D. Gur, and D. M. Livermore. 1998. OXA-16, a further extended-spectrum variant of OXA-10 $\beta$-lactamase, from two Pseudomonas aeruginosa isolates. Antimicrob. Agents Chemother. 42:3117-3122.

7. Danel, F., L. M. Hall, and D. M. Livermore. 1999. Laboratory mutants of OXA-10 $\beta$-lactamase giving ceftazidime resistance in Pseudomonas aeruginosa. J. Antimicrob. Chemother. 43:339-344.

8. Hall, L. M., D. M. Livermore, D. Gur, M. Akova, and H. E. Akalin. 1993. OXA-11, an extended-spectrum variant of OXA-10 (PSE-2) $\beta$-lactamase from Pseudomonas aeruginosa. Antimicrob. Agents Chemother. 37:16371644. 
9. Kohler, T., M. Michea-Hamzehpour, S. F. Epp, and J. C. Pechere. 1999 Carbapenem activities against Pseudomonas aeruginosa: respective contributions of OprD and efflux systems. Antimicrob. Agents Chemother. 43:424427.

10. Li, X. Z., D. M. Livermore, and H. Nikaido. 1994. Role of efflux pump(s) in intrinsic resistance of Pseudomonas aeruginosa: resistance to tetracycline, chloramphenicol, and norfloxacin. Antimicrob. Agents Chemother. 38:17321741

11. Li, X. Z., D. Ma, D. M. Livermore, and H. Nikaido. 1994. Role of efflux pump(s) in intrinsic resistance of Pseudomonas aeruginosa: active efflux as a contributing factor to $\beta$-lactam resistance. Antimicrob. Agents Chemother. 38: $1742-1752$.

12. Livermore, D. M. 2001. Of Pseudomonas, porins, pumps and carbapenems. J. Antimicrob. Chemother. 47:247-250.

13. Livermore, D. M. 1984 . Penicillin-binding proteins, porins and outer-membrane permeability of carbenicillin-resistant and -susceptible strains of Pseudomonas aeruginosa. J. Med. Microbiol. 18:261-270.

14. Livermore, D. M. 1992 . Interplay of impermeability and chromosomal $\beta$-lactamase activity in imipenem-resistant Pseudomonas aeruginosa. Antimicrob. Agents Chemother. 36:2046-2048.

15. Livermore, D. M. 2002. Multiple mechanisms of antimicrobial resistance in Pseudomonas aeruginosa: our worst nightmare? Clin. Infect. Dis. 34:634-640.

16. Livermore, D. M. 2002. The impact of carbapenemases on antimicrobial development and therapy. Curr. Opin. Investig. Drugs 3:218-224.

17. Livermore, D. M., and H. Y. Chen. 1997. Potentiation of $\beta$-lactams against
Pseudomonas aeruginosa strains by Ro 48-1256, a bridged monobactam inhibitor of AmpC $\beta$-lactamases. J. Antimicrob. Chemother. 40:335-343.

18. Livermore, D. M., R. J. Williams, and J. D. Williams. 1981. In-vitro activity of MK0787 ( $N$-formimidoyl thienamycin) against Pseudomonas aeruginosa and other gram-negative organisms and its stability to their $\beta$-lactamases. J. Antimicrob. Chemother. 8:355-362.

19. Masuda, N., E. Sakagawa, S. Ohya, N. Gotoh, H. Tsujimoto, and T. Nishino. 2000. Substrate specificities of MexAB-OprM, MexCD-OprJ, and MexXYOprM efflux pumps in Pseudomonas aeruginosa. Antimicrob. Agents Chemother. 44:3322-3327.

20. National Committee for Clinical Laboratory Standards. 2003. Methods of dilution antimicrobial susceptibility testing of bacteria that grow aerobically, M07-A6. Approved standard, 6th ed. National Committee for Clinical Laboratory Standards, Wayne, Pa..

21. Nikaido, H. 1998. Antibiotic resistance caused by gram-negative multidrug efflux pumps. Clin. Infect. Dis. 27(Suppl. 1):S32-S41.

22. Nordmann, P., and L. Poirel. 2002. Emerging carbapenemases in gram negative aerobes. Clin. Microbiol. Infect. 8:321-331.

23. Tsuji, M., Y. Ishii, A. Ohno, S. Miyazaki, and K. Yamaguchi. 1998. In vitro and in vivo antibacterial activities of S-4661, a new carbapenem. Antimicrob. Agents Chemother. 42:94-99.

24. Yang, Y. J., and D. M. Livermore. 1989. Interactions of meropenem with class I chromosomal $\beta$-lactamases. J. Antimicrob. Chemother. 24(Suppl. A): 207-217. 